\title{
LEIOMIOMA RENAL SINTOMÁTICO: UNA MASA RENAL DE DIFÍCIL DIAGNÓSTICO
}

\author{
L.M. CLEMENTE RAMOS, A. CANDIA FERNÁNDEZ*, A. ALLONA ALMAGRO** \\ Urólogo. Práctica Privada. *Unidad de Anatomía Patológica. Hospital Ruber Internacional. \\ **Servicio de Urología. Hospital Ramón y Cajal.
}

Actas Urol Esp. 27 (7): 546-550, 2003

\section{RESUMEN}

LEIOMIOMA RENAL SINTOMÁTICO: UNA MASA RENAL DE DIFÍCIL DIAGNÓSTICO

El leiomioma renal es un tumor mesenquimatoso benigno poco frecuente que, no obstante, se debe tener en cuenta ante el diagnóstico de una masa renal. Esta neoplasia puede originarse en cualquier órgano del aparato genitourinario que contenga músculo liso, siendo la cápsula renal la localización más frecuente. Dado el comportamiento benigno de la lesión, es subsidiario de la realización de una cirugía renal conservadora. No obstante, ante la dificultad para distinguirlo del adenocarcinoma, no será infrecuente que el diagnóstico se produzca tras el análisis histológico de una pieza de nefrectomía radical.

PALABRAS CLAVE: Leiomioma. Renal. Tumor benigno.

\section{ABSTRACT \\ SYMPTOMATIC LEIOMYOMA OF THE KIDNEY: RENAL MASS WITH DIFFICULT PREOPERATIVE DIAGNOSIS}

Renal leiomyoma is a benign mesenchymal tumour that, albeit very rare, has to be ruled out when a renal mass has been diagnosed. This tumour can arise from any organ of the genitourinary tract with smooth muscle cells, being the renal capsule the most frequent origin. As its behaviour is not aggressive, nephron-sparing surgery is indicated. However, as it is difficult to preoperatively differentiate the leiomyoma from the adenocarcinoma, the former is commonly diagnosed after examination of the entire organ surgically removed.

KEY WORDS: Leiomyoma. Kidney. Benign neoplasm.

$\mathrm{E}$ 1 leiomioma renal se incluye dentro del grupo de los tumores benignos derivados del tejido conjuntivo o mesenquimatoso. Son poco frecuentes, pero deben ser considerados en el diagnóstico diferencial de las masas renales, pues dado el comportamiento benigno de la lesión, son subsidiarios de la realización de una cirugía renal conservadora.

\section{CASO CLÍNICO}

Varón de 59 años sin antecedentes urológicos de interés salvo prostatismo leve (puntuación en cuestionario IPSS 8), que presentaba episodios autolimitados de hematuria monosintomática de un mes de evolución. La exploración física fue anodina. En la ecografía abdominal se apreció la existencia de una masa sólida en el polo inferior 
del riñón izquierdo, homogénea, de 6 x 4,5 cms. La TAC confirmó este hallazgo, evidenciándose una masa sólida en la valva externa de la mitad inferior del riñón izquierdo; se comprobó un realce de la misma tras la infusión de contraste, objetivándose, no obstante, una menor densidad de la misma respecto del resto del parénquima. No se apreciaron datos de enfermedad locoregional o de posible compromiso venoso.

Con el diagnóstico de masa renal derecha probablemente correspondiente a hipernefroma se realizó una nefrectomía radical a través de un abordaje subcostal (hemichevron). No se encontraron hallazgos compatibles con afectación locorregional, procediéndose, no obstante, a la realización de una linfadenectomía regional.

El estudio macroscópico de la pieza quirúrgica mostró un abombamiento de la superficie externa del polo inferior renal producido por una tumoración sólida de $6,5 \mathrm{~cm}$. de diámetro máximo y de color amarillento, bien delimitada, sin extensión extracapsular, de consistencia elástica y superficie de corte arremolinada (Fig. 1). El tumor no rebasaba la cápsula, ni infiltraba la pelvis, la vena renal ni los ganglios linfáticos.

El estudio microscópico evidenció una tumoración bien delimitada, con un patrón de crecimiento fascicular y constituida por células fusiformes, de citoplasmas ligeramente eosinófilos y núcleos ovoides, sin atipia. No se identificaron mitosis, ni necrosis, así como tampoco se demostraron zonas de tejido adiposo ni acumulaciones de vasos anómalos (Fig. 2).

En el estudio inmunohistoquímico, las células se marcaron intensa y difusamente con desmina, vimentina, actina muscular común y de músculo liso. Se comprobó negatividad para CD34, proteína S-100; focalmente se demostró positividad citoplásmica para HMB-45.

El postoperatorio cursó favorablemente, siendo dado de alta en el $6^{-}$día del mismo. Tras un seguimiento de treinta meses, el paciente se encuentra asintomático y libre de enfermedad.

\section{DISCUSIÓN}

El riñón contiene abundante tejido fibroso y conectivo, con músculo liso en la cápsula y alrededor de la pelvis y los cálices. El tejido adiposo está presente en el hilio, y los vasos sanguíneos y

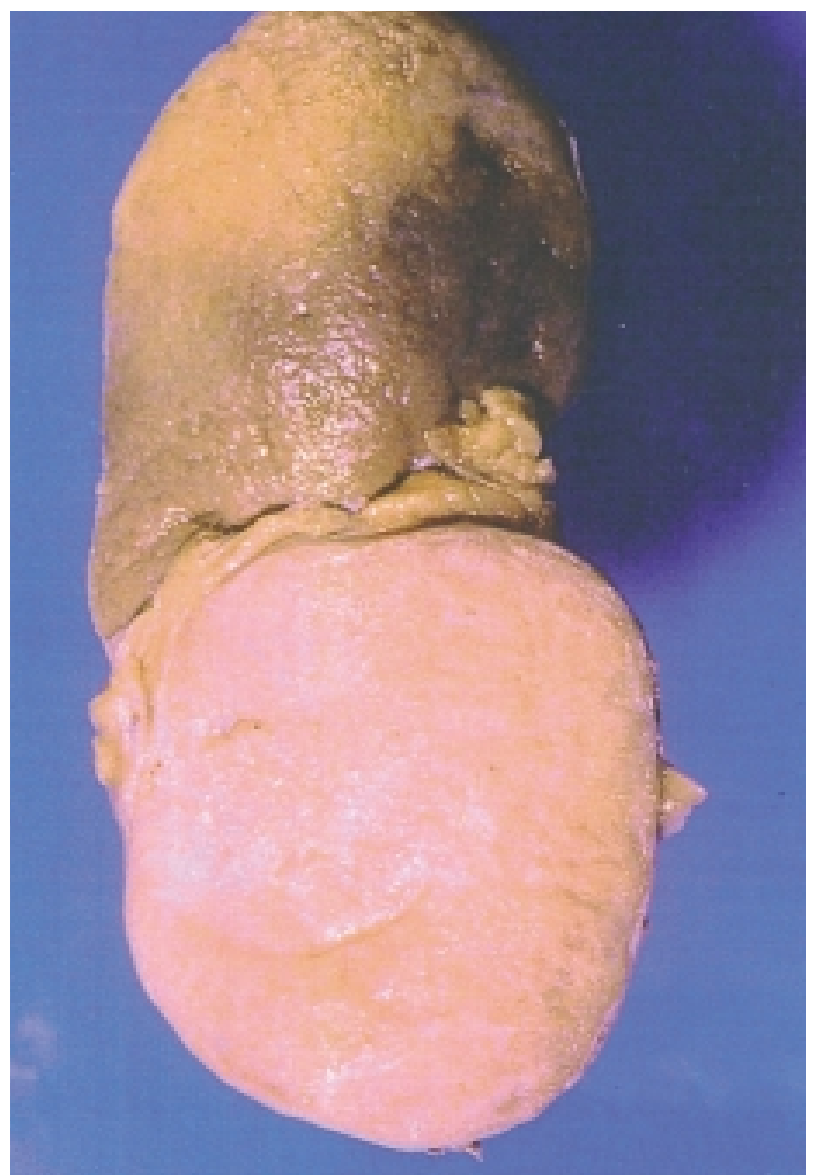

FIGURA 1. La tumoración se localiza en el polo inferior del riñón y es sólida, blanquecina y con una superficie de corte arremolinada.

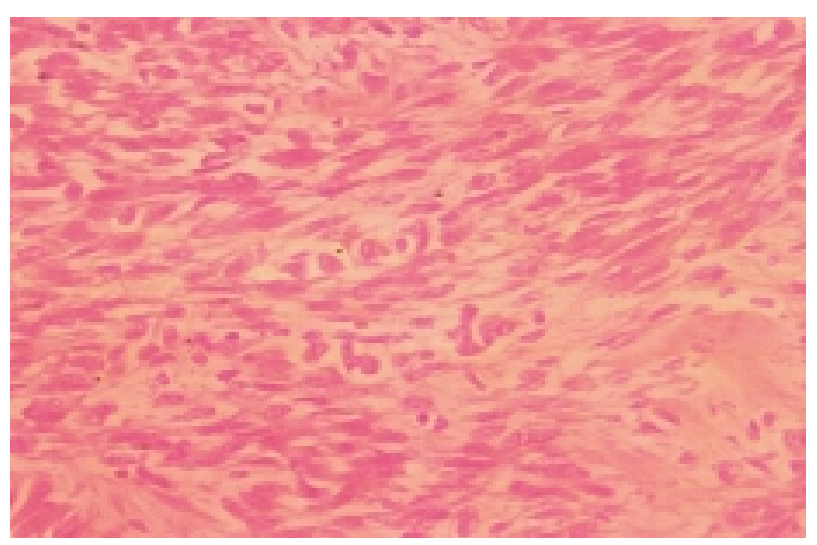

FIGURA 2. El tumor tiene un patrón de crecimiento fascicular y está constituido por células fusiformes sin atipia ni mitosis. HEx20.

los nervios son abundantes. Es factible que puedan aparecer neoplasias desde cualquiera de estos tejidos ${ }^{1}$. El leiomioma se incluye dentro del grupo de los tumores benignos derivados del teji- 
do conjuntivo o mesenquimatoso. Son poco frecuentes, y pueden darse en cualquier órgano del aparato genitourinario que contenga músculo liso. De hecho, se han descrito prácticamente en todas las localizaciones del mismo (la cápsula renal, la pelvis renal, el uréter, la vejiga, la uretra, el cordón espermático, los conductos deferentes, el escroto, las vesículas seminales, el pene y la próstata), siendo la más frecuente en la cápsula renal ${ }^{2,3}$. Se ha estimado que el $90 \%$ de los leiomiomas renales se originan en la cápsula renal, y el 10\% restante en el músculo liso de la pelvis renal. Son más frecuentes en mujeres (66\%) y en pacientes de raza blanca (70\%), siendo más común su hallazgo entre la $2^{\underline{a}}-5^{\underline{a}}$ décadas de la vida. El modo más habitual de presentación es como masa palpable (57\%) y/o dolor en el flanco (53\%). La clásica tríada del carcinoma renal (dolor, masa abdominal y hematuria) se encuentra únicamente en el 3,3\% de los casos ${ }^{4}$. El leiomioma también se ha asociado a la esclerosis tuberosa, si bien las lesiones renales que más frecuentemente acompañan a ésta son los angiomiolipomas y los quistes ${ }^{5}$.

El tumor no presenta predominio de lateralidad, y se localiza en el 74\% de las ocasiones en el tercio inferior del riñón. Suele tener un aspecto sólido (73\%), pudiendo sufrir ocasionalmente una degeneración quística (leiomioma quístico), no siempre relacionada con una degeneración sarcomatosa. La hemorragia aparece en el $17 \%$ de los casos y en un $20 \%$ se encuentran calcificaciones irregulares ${ }^{4,6}$.

- Los de pequeño tamaño (menores de $2 \mathrm{~cm}$ ), múltiples frecuentemente, de localización subcapsular, y que constituyen generalmente hallazgos de autopsia ${ }^{7}$. Es el grupo más frecuente, siendo excepcional su hallazgo clínico ${ }^{8}$.

- Aquellos de gran tamaño, únicos y sintomáticos, que son extremadamente raros, como el presente caso.

- Los que han sufrido degeneración sarcomatosa $^{9}$.

La escasa frecuencia de estos tumores renales no ha permitido describir unos patrones radiológicos específicos, lo cual ha conducido a la realización de cirugía radical en numerosas ocasiones ante el diagnóstico de hipernefroma, ya que frecuentemente es indistinguible radiológicamente del mismo (ECO, TAC y RNM) ${ }^{10-13}$.
Sin embargo, algunos autores indican algunas características que, si bien no son específicas, pueden hacer sospechar la existencia de un leiomioma:

- Patrón sonográfico algo menos refringente que el resto del parénquima renal.

- Lesión bien circunscrita (hipo o hipervascularizada) que no presenta invasión de la vena renal, ni shunt arteriovenosos, ni tampoco invasión de órganos vecinos ni adenopatías. Además, la localización suele ser capsular/subcapsular o peripielica.

- Ausencia de cambios apreciables tras la inyección de epinefrina intra-arterial.

- Ausencia de calcificaciones, aunque algunos autores indican que se pueden objetivar hasta en un $20 \%$ de los casos ${ }^{4,14,15}$.

La T.A.C. es más sensible que la ECO para valorar la procedencia de la lesión, aunque a veces puede resultar difícil diferenciar lo que es de origen primario renal de una infiltración del órgano por una masa de origen retroperitoneal. Habitualmente, la densidad de estas neoplasias está en el rango de los tejidos blandos ${ }^{16}$.

El leiomiosarcoma se origina en las mismas áreas que el leiomioma, siendo clínicamente imposibles de diferenciar los grandes leiomiomas de los leiomiosarcomas, e incluso algunos autores sostienen que estos últimos se producen a partir de la degeneración maligna de aquellos. A menos que exista invasión de otras estructuras, es difícil diferenciar mediante la TAC un leiomiosarcoma de un leiomioma ${ }^{4,17,18}$. Ambos tumores son poco frecuentes; el leiomioma renal es relativamente raro, y el leiomiosarcoma representa el $11 \%$ de todos los tumores retroperitoneales. La presencia de quistes, hemorragia, necrosis y calcificaciones sugieren malignidad ${ }^{19}$. Macroscópicamente, el leiomioma aparece como un tumor sólido, de consistencia elástica al estar formado por músculo y colágeno, bien delimitado, de color blanco-nacarado, blanco-grisáceo o rojizo al corte, dependiendo de la vascularización que presente, y de aspecto fascicular. Característicamente, los tumores benignos se hallan encapsulados $^{20}$. Inmunológicamente, tanto leiomiomas como leiomiosarcomas muestran reactividad para vimentina, actina, miosina muscular lisa, desmina y componentes de la membrana basal, incluyendo laminina y colágeno tipo $4^{21-23}$. 
El diagnóstico diferencial entre leiomioma $\mathrm{y}$ leiomiosarcoma depende de una combinación de características macro y microscópicas. Un índice mitótico elevado es prácticamente diagnóstico de malignidad. El diagnóstico de leiomiosarcoma puede ser sospechado en tumores de gran tamaño, necróticos o hemorrágicos, aunque el número de mitosis sea bajo ${ }^{19}$. Microscópicamente el leiomioma está constituido por haces musculares de fibra lisa, con disposición entrecruzada, constituidos por células fusiformes con mayor o menor cantidad de tejido conectivo (fibromiomas si hay gran cantidad de éste). La presencia de zonas de fibrosis se produce a medida que se incrementa el aporte sanguíneo en el tumor. Sin embargo, no se observan áreas de necrosis. Los núcleos se hallan bien delimitados y no se suelen observar más de diez mitosis/campo ni atipias. La relación nuclear/citoplasmática es baja. La presencia de anormalidades nucleares y un número elevado de mitosis por campo diferencia al leiomioma del leiomiosarcoma ${ }^{2,20,24}$.

La positividad al HMB-45 se ha descrito fundamentalmente en tumores de origen hamartomatoso, como el angiomiolipoma, el rabdomioma y el linfangiomioma. Aunque los estudios al respecto comprenden un escaso número de piezas estudiadas, Bonsib (1996) ${ }^{25}$ observó que las células con positividad al HMB-45 son identificables en varios tumores del músculo liso, en particular aquellos que se originan en la cápsula renal; esta característica no la objetivó en las células sanas de la cápsula renal adyacente ni en los leiomiosarcomas de ese origen. Se podría pensar que las células de estos leiomiomas están relacionadas con la misma célula pluripotencial que da lugar al angiomiolipoma. No obstante, Bonsib revisó cuidadosamente las piezas y no encontró datos que apoyaran el diagnóstico de angiomiolipoma. No obstante, éste es un tema muy controvertido; de hecho otros autores no han encontrado una reactividad al HMB-45 en leiomiomas ${ }^{26}$. En este sentido, Nonomura y cols. (1998) ${ }^{27}$ afirmaron que los angiomiolipomas compuestos casi exclusivamente por células musculares lisas de aspecto fusiforme simulan un leiomioma, por lo que la positividad al HMB-45 de un tumor renal con esas características es prácticamente diagnóstico de angiomiolipoma, si bien esta afirmación se basaba, fundamentalmente, en análisis de masas hepáticas.
Es conocida la asociación entre el virus de Epstein-Barr y la presencia de diferentes tumores en pacientes inmunodeprimidos, y entre ellos, los tumores del músculo liso. Estos han sido descritos en diversos órganos, entre ellos el riñón. Por ello, el leiomioma debe tenerse en cuenta a la hora de plantear el diagnóstico diferencial de una masa renal en este subgrupo de población ${ }^{28}$.

El pronóstico de este tumor es excelente ${ }^{4}$, si bien excepcionalmente se ha constatado la metastatización que, al igual que el tumor primitivo, conservan su aspecto histológico benigno 29,30 .

A pesar de su baja frecuencia, el leiomioma renal sintomático (único) debe ser considerado en el diagnóstico diferencial de las masas renales. Dado el comportamiento benigno de la lesión, es obligado tener presente la posibilidad de estos tumores al valorar una masa renal, y máxime ante lesiones que presenten las características radiológicas descritas y/o pacientes inmunodeprimidos. Dado que la cirugía preservadora de nefronas en el tratamiento del carcinoma unilateral de células renales es ciertamente una alternativa factible a la nefrectomía radical en pacientes con tumores de diámetro menor a $4 \mathrm{~cm}$., podríamos encontrarnos con el diagnóstico de leiomioma o de angiomiolipoma en la pieza procedente de esta cirugía parcial si la aplicamos este criterio de entrada a cualquier masa que no sobrepase esas dimensiones. Si decidimos realizar una biopsia intraoperatoria, independientemente del tamaño del tumor, y se confirma la estirpe tumoral benigna, es preceptiva la realización de una cirugía renal conservadora. No obstante, considerando la dificultad que implica la sospecha clínica de estas neoplasias, no será infrecuente que el diagnóstico se produzca tras el análisis histológico de una pieza de nefrectomía radical $^{31,32}$.

\section{REFERENCIAS}

1. JENKINS JD. Renal sarcomas. Br J Urol 1971; 43: 263.

2. TALLADA M, MARTÍNEZ A.: Leiomioma vesical. En: Patología excepcional en Urología. Resel L, Leiva O, Burgos J, Díaz R, Llorente C (eds). Madrid, Luzán 5 Eds 1998: 123-140.

3. BELIS JH, POST GJ, ROCHMAN SC et al.: Genitourinary leiomyomas. Urology 1979; 13: 424. 
4. STEINER M, QUINLAN D, GOLDMAN $\mathrm{S}$ et al.: Leiomyoma of the kidney: presentation of 4 new cases and the role of computerized tomography. $J$ Urol 1990; 143: 994-998.

5. TSUJIMURA A, MIKI T, SUGAO $\mathrm{H}$ et al.: Urol Int 1996; 57: 192-193.

6. RABADE CJ, FERNÁNDEZ JM, ALVAREZ S et al.: Leiomioma renal. Aportación de un nuevo caso. Actas Urol Esp 1994; 18: 816-818.

7. XIPEL JM.: The incidence of benign renal nodules (a clinicopathologic study). J Urol 1971; 106: 503.

8. WITTEN D, MYERS G, UTZ D.: Tumores del aparato genitourinario en urografía clínica de Emmet. Atlas y tratado roentgenológico, 4⿳亠丷厂 Ed. Barcelona. Ed. Salvat 1983; 3: 103-404.

9. MOHAMMED AY, MATTHEW L, HARMSE JL et al.: Multiple leiomyoma of the renal capsule. Scand $J$ Nephrol 1999; 33: 138-139.

10. MOHLER JL, CASALE AS.: Renal leiomyoma. J Urol 1987; 138: 853.

11. MOUNIC V, PAILLET C, FEIL C et al.: Leiomyome renal. J d'Urol 1989; 4: 239.

12. MOHAMMED AY, MATTHEW L, HARMSE JL et al.: Multiple leiomyoma of the renal capsule. Scand $J$ Nephrol 1999; 33: 138-139.

13. CORTADELLAS A, CASTELLANOS RI, GUZMÁN A et al.: Leiomioma de cápsula renal. Presentación de un caso y revisión de la literatura. Arch Esp Urol 1992; 45: 478-480.

14. COSTELLO P, ZINMAN L, MICHAUD J.: The angiography of intrarenal leiomyoma. J Can Assoc Radiol 1978; 29: 134.

15. PALMER FJ Y TYNAM AP. Leiomyoma of the kidney. J Urol 1974; 112: 22.

16. SHIRKODA A, LEWIS E.: Renal sarcoma and sarcomatoid renal cell carcinoma: CT and angiographic features. Radiology 1987; 162: 353.

17. ROY C, PFLEGER D, TUCHMAN C et al.: Small leiomyosarcoma of the renal capsule: CT findings. Eur Radiol 1998; 8: 224-227.

18. RIQUET H, FABRE B, SKOWRON O, et al: Report of a case of leiomyoma of the kidney. Prog Urol 2002 Feb 12 (11): 42-45.

19. VICENTE J, VAYREDA JM.: Masa renal con afectación regional "avanzada". En: Patología excepcional en Urología. Resel L, Leiva O, Burgos J, Díaz R, Llorente C (eds). Madrid, Luzán 5 Eds, 1998: 281309.

20. LAKE MH, KOSOW AS, BOKINSKY G.: Leiomyoma of the bladder and urethra. J Urol 1981; 125: 742743 .
21. CHOW LC.: Fine needle aspiration cytodiagnosis of leiomyosarcoma of the renal pelvis. Acta Cytol 1994; 38: 759 .

22. RO JY.: Sarcomatoid renal cell carcinoma: clinicopathology. Cancer 1987; 59: 516.

23. VILLANUEVA RR.: Leiomyosarcoma of the kidney. Report of a case diagnosed by fine needle aspiration cytology and electron microscopy. Acta Cytol 1994; 38: 568.

24. CATALONA WJ.: Tumores uroteliales del tracto urinario. En: Walsh PC, Retik AB, Stamey TA, Vaughan ED. Campbell's Urology. Ed. Panamericana 1994: 1091-1133.

25. BONSIB SM.: HMB-45 reactivity in renal leiomyomas and leiomyosarcomas. Mod Pathol 1996; 9: 664-669.

26. ASHFAQ R, WEINBERG AG, ALBORES-SAAVEDRA J.: Renal angiomiolypomas and HMB reactivity. Cancer 1993; 71: 3091.

27. NONOMURA A, MINATO H, KURUMAYA H.: Angiomyolipoma predominantly composed of smooth muscle cells: problems in histological diagnosis. Histopathology 1998; 33: 20-27.

28. KRISHNAN R, FREEMAN JA, CREAGER AJ.: Epstein-Barr virus induced renal leiomyoma. $\mathrm{J}$ Urol 1999; 161: 212.

29. CORROY J, PEILLERON B, SIX A et al.: Le leiomyome pedicule du rein. A propos d'un cas. J d’Urol 1989; 95: 72.

30. MONTOYA MD, GARCÍA PJ, GUTIÉRREZ JM et al.: El leiomioma renal sintomático. Arch Esp Urol 1993; 46: 833-835.

31. BUTLER BP, NOVICK AC, MILER DP et al.: Management of small unilateral renal cell carcinomas: radical versus nephron-sparing surgery. Urology 1995; 13: 288-291.

32. LERNER SE, HAWINS CA, BLUTE ML et al.: Disease outcome in patients with low stage renal cell carcinoma treated with nephron sparing or radical surgery. J Urol 1996; 155: 1868-1873.

Dr. L.M. Clemente Ramos

Unidad de Urología

Hospital Ruber Internacional

Avda. España, 3 - Esc. 2, 1 5

28220 Majadahonda (Madrid)

(Trabajo recibido el 15 abril de 2002) 